\title{
Rapid Nicotine Clearance is Associated with Greater Reward and Heart Rate Increases from Intravenous Nicotine
}

\author{
Mehmet Sofuoglu*', Aryeh I Herman', Haleh Nadim² and Peter Jatlow ${ }^{2}$ \\ 'Department of Psychiatry and VA Connecticut Healthcare System, Yale University School of Medicine, West Haven, CT, USA; ${ }^{2}$ Department of \\ Laboratory Medicine, New Haven, CT, USA
}

\begin{abstract}
The ratio of nicotine metabolites (trans-3'-hydroxycotinine $(3 \mathrm{HC})$ to cotinine) correlates with nicotine clearance. In previous studies, high nicotine metabolite ratio (NMR) predicted poor outcomes for smoking cessation treatment with nicotine patch. The underlying mechanisms that associate NMR with treatment outcomes have not been fully elucidated. A total of 100 smokers were divided into quartiles based on their baseline plasma NMR. Following overnight abstinence, smokers received saline followed by escalating intravenous doses of nicotine $(0.5$ and $1.0 \mathrm{mg} / 70 \mathrm{~kg})$ given $30 \mathrm{~min}$ apart. The effects of nicotine on subjective, plasma cortisol, heart rate, and systolic and diastolic blood pressure measures were obtained. Smokers in the first NMR quartile (slower metabolizers) had lower Fagerstrom Test for Nicotine Dependence (FTND) scores, suggesting lower levels of dependence. In contrast, smokers in the fourth NMR quartile (faster metabolizers) reported greater craving for cigarettes following overnight abstinence from smoking and reported greater ratings of nicotine-induced good drug effects, drug liking, and wanting more drug. Higher NMR was also associated with greater heart rate increases in response to nicotine. These results suggest that enhanced nicotine reward and cigarette craving may contribute to the poor treatment response in smokers with high NMR. These findings warrant further investigation, especially in treatment-seeking smokers undergoing cessation treatment.

Neuropsychopharmacology (2012) 37, 1509-1516; do::I0.1038/npp.2011.336; published online 15 February 2012
\end{abstract}

Keywords: nicotine dependence; intravenous nicotine; nicotine abstinence

\section{INTRODUCTION}

Nicotine is considered to be the main psychoactive and addictive component of tobacco. It is inactivated in the liver to its primary metabolite, cotinine, primarily by the CYP2A6 microsomal enzyme (Benowitz et al, 2009; Hukkanen et al, 2005). Although this is the major pathway for nicotine elimination, it is not the only route (Benowitz et al, 2009; Hukkanen et al, 2005). The same enzyme oxidizes cotinine to $3^{\prime}$-hydroxycotinine $(3 \mathrm{HC})$. The gene encoding the CYP2A6 enzyme is highly polymorphic, and multiple functional allelic variations and SNPs have been described (Mwenifumbo and Tyndale, 2009). In addition, many environmental factors regulate CYP2A6 activity, thereby affecting the rate of nicotine metabolism (Benowitz et al, 2009). Given that multiple genetic and non-genetic factors influence CYP2A6 activity, a phenotypic marker

*Correspondence: Dr M Sofuoglu, Department of Psychiatry and VA Connecticut Healthcare System, Yale University School of Medicine, 950 Campbell Ave., Bldg. 36/II6A4, West Haven, CT 06516, USA, Tel: + I (203) 937-4809, Fax: + I (203) 937-3478,

E-mail: Mehmet.Sofuoglu@yale.edu

Received 30 August 2011; revised 16 December 2011; accepted 20 December 2011 reflecting the rate of nicotine metabolism is needed. Many studies have convincingly shown that the ratio of plasma $3 \mathrm{HC} /$ cotinine is one such marker (Benowitz and Jacob, 2001; Benowitz et al, 2003; Dempsey et al, 2004). Because the nicotine metabolite ratio (NMR) correlates with nicotine clearance and CYP2A6 genotype (Benowitz et al, 2006a), it is considered to be a marker of CYP2A6 activity (Dempsey et al, 2004). The NMR is stable over time because of relatively long half-life of cotinine (16h) (Lea et al, 2006; Mooney et al, 2008). In contrast, the metabolic ratio of cotinine/nicotine is sensitive to the interval since the last exposure to smoking because of the short half life of nicotine (1-2h). The NMR accounts for approximately $69 \%$ of nicotine's oral clearance (Dempsey et al, 2004), although this has been reported to improve with use of the natural logarithm of the ratio (Levi et al, 2007). The NMR is influenced by many variables, including genetic variation, sex, race, and body mass index (BMI) (Hukkanen et al, 2005; Johnstone et al, 2006; Kandel et al, 2007; Swan et al, 2009). The clinical correlations of the NMR have been examined in several studies (Malaiyandi et al, 2006). Although the NMR did not consistently correlate with nicotine dependence or cigarette consumption (for a review see (West et al, 2011)), it did correlate with withdrawal 
severity in adolescent smokers (Rubinstein et al, 2008) and higher craving in smokers using nicotine patch (Lerman et al, 2006). A recent study suggested that the NMR might influence smoking behavior; smokers with higher NMR had greater puff volumes and increased levels of carcinogen biomarker (Strasser et al, 2011). These findings are consistent with previous studies indicating that smokers maintain plasma nicotine levels by adjusting their smoking behavior (Benowitz et al, 1998; Dallery et al, 2003); those with more rapid nicotine clearance would be expected to have greater nicotine intake than those with slower disposition.

The NMR ratio may also be predictive of smoking cessation outcomes. Smokers in the lowest NMR quartile (slower metabolizers), compared with those in higher quartiles, respond better to nicotine patch for smoking cessation (Schnoll et al, 2009). In another clinical trial that tested the efficacy of bupropion for smoking cessation, smokers in the fourth NMR quartile (faster metabolizers) had poor smoking cessation rates if they were assigned the placebo treatment. However, bupropion offset the relapse liability among high NMR smokers (Patterson et al, 2008). Overall, these findings are consistent with the idea that smokers who rapidly metabolize nicotine might be more dependent and have greater difficulty with smoking cessation. How rapid nicotine metabolism moderates smoking behavior or treatment response is not well understood, but it might enhance withdrawal severity or result in greater sensitivity to nicotine reinforcement (Benowitz et al, 2009). To address these possibilities, we examined the influence of NMR on acute response to nicotine in overnight abstinent smokers. We used intravenous (IV) nicotine administration, which rapidly delivers precise doses of nicotine, comparable to the bolus effect of smoking. These features of IV administration allow the assessment of reward and other acute effects of nicotine (Sofuoglu et al, 2008). Outcome measures included subjective measures, heart rate, blood pressure, plasma cortisol, and nicotine-withdrawal symptoms.

\section{MATERIALS AND METHODS}

\section{Participants}

In total, 107 non-treatment-seeking smokers were recruited from the New Haven, Connecticut area for a study examining the genetic predictors of nicotine reward. Potential participants were between the ages of 18 and 50 and smoked at least 10 cigarettes/day. Seven participants were not included in the final analysis because of low cotinine levels $(<10 \mathrm{ng} / \mathrm{ml})$ that reduced NMR measurement reliability (Kandel et al, 2007). Characteristics of the 100 smokers included in the analysis are shown in Table 1. Participants were medically healthy volunteers who were not dependent on alcohol or drugs other than nicotine, as determined by the Structured Clinical Interview for DSM-IV (First et al, 1995) and verified by urine drug screening. Written consent was provided before participating in the study. Experimental sessions were conducted in the Biostudies Unit located at the VA Connecticut Healthcare System, and the participants were paid for their participation. This study was approved by the VA Connecticut Healthcare System Human Subjects Subcommittee.

Table I Baseline Measures for the Study Sample

\begin{tabular}{|c|c|c|c|c|c|c|}
\hline Measures & Overall & Ist Quartile & 2nd Quartile & 3rd Quartile & 4th Quartile & $P^{\mathbf{a}}$ \\
\hline Age, years & $37.0(8.9)$ & $36.7(8.8)$ & $38.5(9.3)$ & $36.2(9.2)$ & $36.6(8.9)$ & NS \\
\hline Sex, Female, $n$ & 27 & 6 & 8 & 6 & 7 & NS \\
\hline \multicolumn{7}{|l|}{ Race, $n$} \\
\hline African-American & 47 & 14 & 12 & 11 & 10 & NS \\
\hline Body mass index & $28.9(5.3)$ & $29.7(4.8)$ & $27.9(5.1)$ & $28.7(4.6)$ & $29.1(6.6)$ & NS \\
\hline FTND & $5.5(2.2)$ & $4.6(2.2)$ & $5.8(2.3)$ & $6.1(2.2)$ & $5.7(1.7)$ & 0.05 \\
\hline Cigarette consumption/day & I7.7 (8.5) & I6.4 (7.4) & | $8.4(8.3)$ & $19.7(10.8)$ & I $6.7(6.9)$ & NS \\
\hline Duration of smoking, years & $16.8(4.6)$ & 17.6 (4.8) & $17.2(4.3)$ & | $6.3(3.8)$ & |6.| (5.4) & NS \\
\hline Cotinine, ng/ml & $230.9(|4| .2)$ & $265.8(158.3)$ & $276.3(123.2)$ & $238.4(150.3)$ & | $43.4(89.1)$ & $<0.0001$ \\
\hline Nicotine & $2.9(3.6)$ & $4.3(3.2)$ & $3.7(3.6)$ & $2.7(4.3)$ & ।.7 (2.7) & 0.05 \\
\hline MNWS score & $21.5(0.9)$ & $25.2(4.2)$ & $22.4(3.8)$ & $24.1(3.8)$ & $27.3(4.1)$ & NS \\
\hline Craving for cigarettes & $5.1(0.2)$ & $5.4(0.7)$ & $5.9(0.6)$ & $6.6(0.6)$ & $7.2(0.7)$ & $<0.05$ \\
\hline
\end{tabular}

Abbreviations: FTND, Fagerstrom Test for Nicotine Dependence; MNWS, Minnesota Nicotine Withdrawal Scale.

${ }^{a} P$ values indicate significant group differences $P<0.05$.

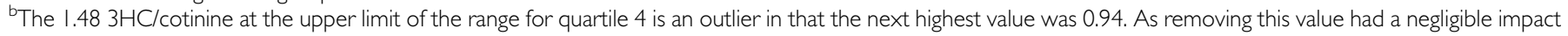
on the study findings, it was included in the final analysis. 


\section{Procedures}

We utilized a single-blind study design. Following overnight abstinence from smoking, participants arrived at the outpatient clinic at approximately $8 \mathrm{AM}$ for the experimental session. Abstinence from smoking was verified with expired carbon monoxide ( $\mathrm{CO} ;<10$ parts-per-million). Participants were asked to fast after midnight to minimize the influence of food on nicotine responses. They were instructed to continue their usual caffeine intake to prevent caffeine withdrawal. A urine screening was performed immediately before the session to rule out recent drug use that excluded subjects from the study. An indwelling catheter was placed in an antecubital vein, and baseline measures were collected. Then, smokers received IV saline followed by two increasing doses of IV nicotine (0.5, and $1.0 \mathrm{mg} / 70 \mathrm{~kg}$ ). Our prior work demonstrated that these doses produced robust physiological and subjective responses in male and female smokers (Sofuoglu et al, 2006). The injections were given $30 \mathrm{~min}$ apart, which allowed subjective and cardiovascular responses to return to baseline levels (Sofuoglu et al, 2005; Sofuoglu et al, 2006; Sofuoglu et al, 2009). An escalating dosing schedule rather than counterbalanced dosing was chosen to prevent any carryover effects from the preceding nicotine dose to saline and to increase the safety of the study by exposing subjects first to saline, followed by a low dose of nicotine before a higher dose was administered.

\section{Drugs}

Nicotine administration. Nicotine bitartrate was acquired from Interchem Corporation (Manchester, Connecticut). All nicotine samples were prepared by a research pharmacist at the VA CT Healthcare System. A total volume of $5 \mathrm{ml}$, containing either $0.5 \mathrm{mg}$ or $1 \mathrm{mg} / 70 \mathrm{~kg}$ of nicotine, was injected over $30 \mathrm{~s}$ intravenously via a catheter located in a forearm vein.

Outcome measures. Our outcome measures assessed biochemical, physiological, and subjective domains. Biochemical measures included $\mathrm{CO}$, plasma nicotine, cotinine, 3HC, and cortisol levels. Expired CO and plasma nicotine concentrations were used to verify overnight abstinence from smoking. Plasma cotinine concentrations were used as a measure of prior nicotine exposure (Benowitz et al, 2002). Expired CO, plasma nicotine, $3 \mathrm{HC}$, and cotinine measurements were taken before the experimental session. Plasma cortisol measurements were taken at baseline, before each of three injections, and at the end of the session. Plasma cortisol has been shown to be a sensitive measure of nicotine withdrawal and administration (al'Absi et al, 2002; Mendelson et al, 2005; Newhouse et al, 1990; Pickworth and Fant, 1998). Physiological measures included systolic and diastolic blood pressure and heart rate, which were measured daily during medication treatment and were taken in the experimental sessions at $-5,1,2,3,5,8,10$, and $15 \mathrm{~min}$ in relation to saline or nicotine deliveries. The subjective measures included the Drug Effects Questionnaire (DEQ) and the Minnesota Nicotine Withdrawal Scale (MNWS). The DEQ was used to measure acute effects from IV nicotine and consisted of seven items: drug strength, high, feel stimulated, good effects, bad effects, want more drugs, and like the drug. Smokers rated each item on a $100 \mathrm{~mm}$ scale, from 'not at all' to 'extremely.' The DEQ was given $1,3,5,8,10$, and $15 \mathrm{~min}$ after saline and nicotine administration. The MNWS measures withdrawal symptoms from tobacco, and includes items to assess cigarette craving, irritability/anger, anxiety, difficulty concentrating, restlessness, increased appetite, depressed or sad mood, and insomnia (Hughes and Hatsukami, 1986; Hughes and Hatsukami, 1997). The MNWS was administered before and after the session.

Assays. Unconjugated plasma cotinine and 3HC concentrations were assayed utilizing HPLC interfaced with tandem mass spectrometry (LC/MS/MS) with stable isotope (deuterated)-labeled internal standards. The assay was similar, but not identical, to that described in the literature (Dempsey et al, 2004). 3HC and 3HC-D3 were purchased from Toronto Research Chemicals, (Ontario, Canada); cotinine, cotinine-D3, nicotine and nicotine-D4 were purchased from Cerilliant, Round Rock, TX. All primary standards and controls were prepared in human plasma determined to be free of nicotine and cotinine. Assays were performed in a building in which tobacco use was not allowed. Separate ion transitions were used for purposes of quantitation and confirmation for each of the three analytes as follows: (1) 3HC: $193 \rightarrow 80$, quantifier: $193 \rightarrow 134$; qualifier, 3HC-D3 $196 \rightarrow 80$ internal standard; (2) cotinine: $177 \rightarrow 80$, quantifier $177 \rightarrow 98$; qualifier: cotinine-D3 $180 \rightarrow 80$ internal standard; (3) nicotine: $163 \rightarrow 132$; quantifier, $163 \rightarrow 106$; qualifier, nicotine-D4 $167 \rightarrow 136$ internal standard.

The cotinine/3HC assay utilized a plasma 'crashing' (zinc sulfate protein precipitation/methanol extraction) technique before LC/MS/MS; organic solvent extraction followed by aqueous micro back-extraction was used to prepare the samples for the nicotine assay. Cotinine was also assayed with this more sensitive solvent extraction procedure, which is capable of measuring concentrations well below $1 \mathrm{ng} / \mathrm{ml}$. However, for determination of baseline $3 \mathrm{HC} /$ cotinine ratios in smokers, the cotinine/3HC sample preparation procedure was used for both metabolites. Cotinine values by the two sample preparation procedures were virtually identical $(r>0.99)$. Between-day coefficients of variation (CVs) over the range of values observed in this study were 6.6 to $8.1 \%$ for cotinine and 4.4 to $5.9 \%$ for $3 \mathrm{HC}$, respectively. Betweenday CVs for nicotine were 8.6 to $13.4 \%$, the latter for a nicotine concentration of $1 \mathrm{ng} / \mathrm{ml}$. More sensitive, but more labor-intensive, procedures for $3 \mathrm{HC}$ and cotinine have been published that are suitable for detecting sub-nanogram concentrations that occur following second-hand smoke exposure (Jacob et al, 2011); however, the current procedure is more than adequately sensitive for assaying samples from smokers.

Data analysis. Study outcomes were analyzed with a repeated-measures model that included both within- and between-subject factors (mixed model) using the Statistical Analysis System, version 9.1.3. (SAS Institute Inc., 2007). The model included the NMR quartile (1 to 4 ) as a fixed effect. For blood pressure, heart rate, and DEQ measurements, where multiple measurements were collected for 
each saline and each nicotine dose, we included the maximum post-dose measure for each dose in our analyses. For these outcomes, the model included fixed main effect terms for dose (saline, $0.5 \mathrm{mg} / 70 \mathrm{~kg}$ nicotine, or $1 \mathrm{mg} / 70 \mathrm{~kg}$ nicotine). Variables that have been shown to influence NMR (sex, race, age, and BMI) were included as covariates in our model. Baseline demographic and smoking measures were compared with analysis of variance (ANOVA) and $\chi^{2}$ tests. Values of $P \leqslant 0.05$ in two-tailed tests were considered statistically significant unless otherwise specified. Significant main effects for group were followed up by post hoc comparisons of groups for significant differences, with Tukey adjustments to prevent Type I errors.

\section{RESULTS}

\section{Baseline Variables}

Baseline variables for four quartiles are shown in Table 1. Smokers in the fourth quartile had significantly lower baseline plasma nicotine (main effect for group: $F(3,96)=5.2 ; P=0.05$ ) and cotinine levels (main effect for group: $F(3,96)=5.2 ; P<0.01)$ than those in other quartiles. The first quartile was associated with lower Fagerstrom Test for Nicotine Dependence (FTND) scores than the second and third quartiles (main effect for group: $F(3,96)=2.7$; $P=0.05)$. Those in the fourth quartile had significantly higher scores than those in the first and third quartile for the first FTND item 'How soon after you wake up do you smoke your first cigarette?' (main effect for group: $F(3,96)=2.8 ; P<0.05)$. The fourth quartile was associated with greater craving for cigarettes than the first and second quartiles (main effect for group: $F(3,96)=5.2 ; P<0.01$ ) (Figure 3). For other measures, there were no significant group differences.

\section{Physiological Response to IV Nicotine}

Both 0.5 and $1 \mathrm{mg} / 70 \mathrm{~kg}$ nicotine doses increased heart rate (main effect for dose: $F(2,186)=239.7 ; P<0.0001$ ) as well as systolic (main effect for dose: $F(2,186)=17.1 ; P<0.0001$ ) and diastolic (main effect for dose: $F(2,186)=9.7$; $P<0.0001)$ blood pressures compared with saline. The responses were dose-dependent such that the $1 \mathrm{mg} / 70 \mathrm{~kg}$ nicotine led to greater responses than $0.5 \mathrm{mg} / 70 \mathrm{~kg}$ for all three outcomes $(P<0.0001)$. Following nicotine administration, peak heart rate and blood pressure values were reached at $1 \mathrm{~min}$ and returned close to baseline values within $30 \mathrm{~min}$ (Figure 1). Those in the fourth quartile had greater heart rate responses to $0.5 \mathrm{mg} / 70 \mathrm{~kg}$ nicotine administration than those in the first quartile (main effect for dose-by-group interaction: $F(6190)=$ $2.6 ; P<0.05)$. There were no significant group differences $(P>0.05)$ for systolic and diastolic blood pressure measurements.

\section{Subjective Responses to IV Nicotine}

Both 0.5 and $1 \mathrm{mg} / 70 \mathrm{~kg}$ nicotine increased the ratings of stimulation (main effect for dose: $F(2,191)=26.7$; $P<0.0001$ ), high (main effect for dose: $F(2,191)=26.0$; $P<0.0001$ ), feel good effects (main effect for dose:
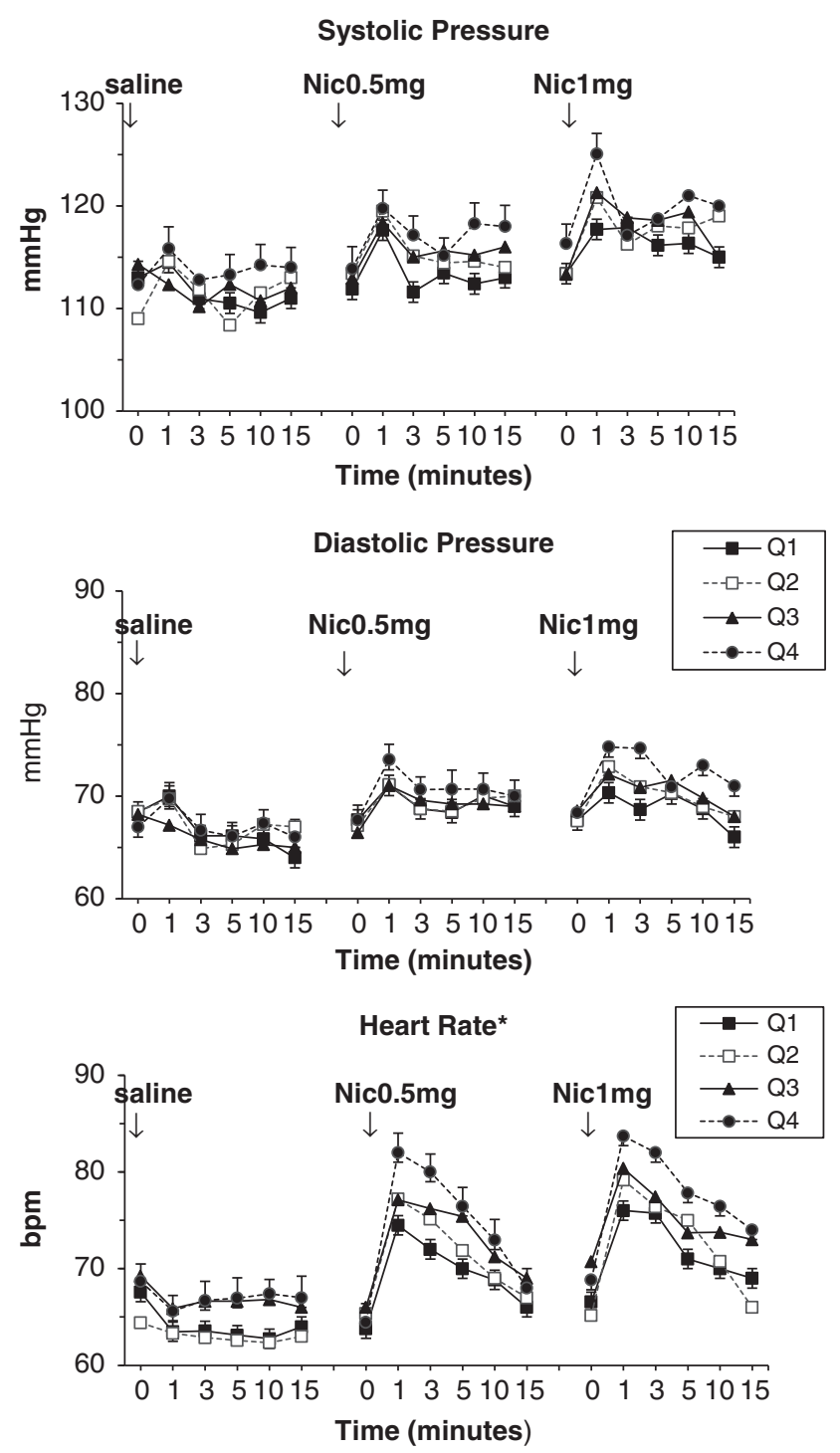

Figure I The influence of nicotine metabolism rate on average ( \pm SEM) heart rate as well as systolic and diastolic pressure responses to saline, 0.5 , and $1.0 \mathrm{mg} / 70 \mathrm{~kg}$ IV nicotine administration. Smokers were divided into quartiles based on their $3 \mathrm{HC} /$ cotinine ratios, which reflect the rate of nicotine metabolism. The doses were given in an ascending order, $30 \mathrm{~min}$ apart. Measurements were taken just before and I, 2 3, 5, 8, 10, 15 and $25 \mathrm{~min}$ after dose delivery. Some of the time-point error bars are not shown for clarity. * Indicates significant group differences $p<0.05$.

$F(2,191)=16.7 ; P<0.0001)$, like the drug effects (main effect for dose: $F(2,191)=16.7 ; P<0.0001)$, want more drugs (main effect for dose: $F(2,191)=12.3 ; P<0.0001$ ), feel bad effects (main effect for dose: $F(2,191)=5.5 ; P<0.01$ ) and feel the drug strength (main effect for dose: $F(2,191)=44.9 ; P<0.0001)$ compared with saline. For all these items, the ratings under 0.5 or $1.0 \mathrm{mg} / 70 \mathrm{~kg}$ of nicotine were greater than those under placebo $(P<0.05)$. For the rating of stimulation, responses under $1 \mathrm{mg} / 70 \mathrm{~kg}$ were greater than those for $0.5 \mathrm{mg} / 70 \mathrm{~kg}$ nicotine $(P<0.01)$. Following nicotine administration, subjective responses were reached within $1 \mathrm{~min}$ and returned close to baseline values within $10 \mathrm{~min}$ (Figure 2).

Those in the fourth quartile had greater responses than those in the first quartile for the rating of good drug effects 

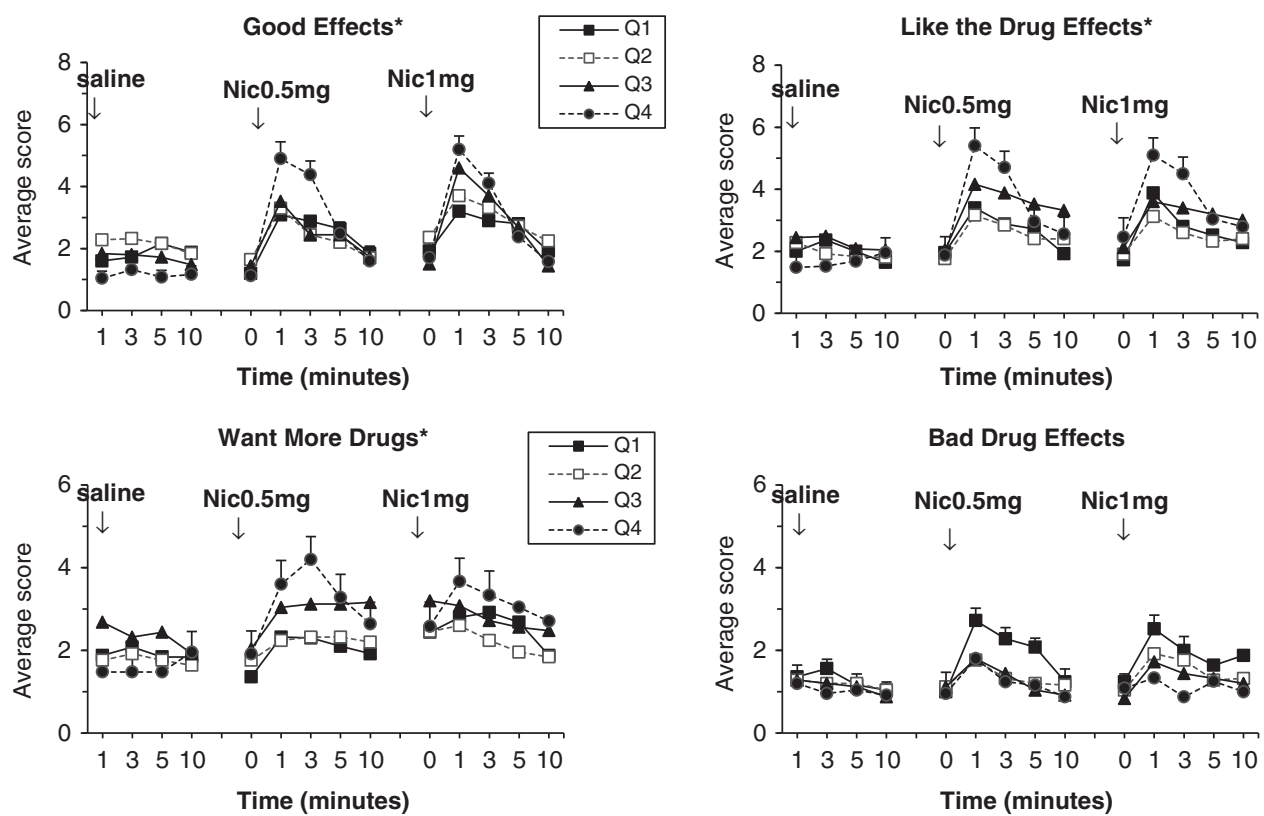

Figure 2 The influence of nicotine metabolism rate on selected average ( \pm SEM) subjective responses to saline, 0.5 , and $1.0 \mathrm{mg} / 70 \mathrm{~kg} \mathrm{IV}$ nicotine administration. The doses were given in an ascending order and 30 min apart. Measurements were taken just before and I, 3, 5, 8, I0, I5 and 25 min after dose delivery. Some of the time-point error bars are not shown for clarity. *Indicates significant group differences $p<0.05$.

in response to $0.5 \mathrm{mg} / 70 \mathrm{~kg}$ nicotine dose (main effect for dose-by-group interaction: $F(6,191)=3.1 ; \quad P<0.01)$. Similarly, for like drug effects, smokers in the fourth quartile had greater ratings than those in the first quartile in response to both doses of nicotine (main effect for dose-by-group interaction: $F(6,191)=2.9 ; P<0.01)$. For the rating of want more drugs, smokers in the fourth quartile had greater responses than those in the first and second quartiles in response to the $0.5 \mathrm{mg} / 70 \mathrm{~kg}$ (main effect for dose-by-group interaction: $F(6,191)=2.7$; $P<0.05)$.

\section{Nicotine Withdrawal Symptoms}

Smokers in the fourth quartile had significantly greater cigarette craving than those in the first quartile (main effect for group: $F(3,93.8)=3.1 ; P<0.05)$ (Figure 3). For the total MNWS score, no group differences were observed in response to nicotine challenge.

\section{DISCUSSION}

Similar to previous studies, we divided our subjects into quartiles on the basis of NMR. The average NMR values for our study's quartiles were comparable to those reported previously (Schnoll et al, 2009; Strasser et al, 2011). We found that smokers in the fourth quartile (faster metabolizers) reported greater craving for cigarettes following overnight abstinence from smoking. In contrast, smokers in the first quartile (slower metabolizers) had lower FTND scores, suggesting lower levels of dependence. Furthermore, higher NMR was associated with greater nicotine-induced reward and increased heart rate in overnight abstinent smokers.

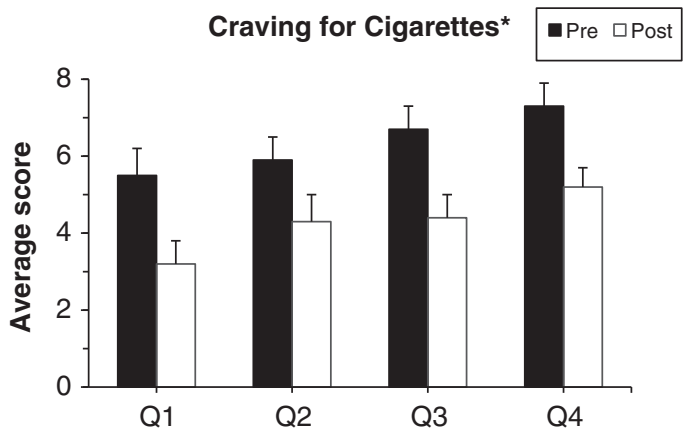

Figure 3 The influence of nicotine metabolism rate on average ( \pm SEM) cigarette craving. The measurements were obtained at the beginning and end of the session. *Indicates significant group differences $p<0.05$.

The association of lower FTND scores with lower NMR is consistent with previous reports suggesting that smokers with slower nicotine metabolism may be less-dependent than those with faster metabolism (Mwenifumbo et al, 2007; Rao et al, 2000; Wassenaar et al, 2011). Although other studies did not find an association between NMR and the level of nicotine dependence (Johnstone et al, 2006), smokers with faster nicotine metabolism reported greater craving for cigarettes following overnight abstinence from smoking. However, the groups did not differ with respect to overall nicotine withdrawal severity as assessed by the MNWS. As mentioned above, previous studies have found that smokers with high NMR had greater nicotine withdrawal severity and craving for cigarettes (Lerman et al, 2006; Rubinstein et al, 2008), although others did not find an association between NMR and craving or withdrawal (Patterson et al, 2008; Schnoll et al, 2009). The reasons for these conflicting findings remain to be determined. 
In our study, higher NMR was associated with lower plasma nicotine and cotinine levels following overnight abstinence from smoking. Although the lower nicotine finding may reflect more rapid clearance of nicotine in association with the higher NMR (Dempsey et al, 2004), considering the short half life of nicotine, it is not possible to draw any definitive conclusions without knowing the time of the last nicotine exposure. The low cotinine levels in the high NMR group perhaps reflect a greater sensitivity of cotinine to changes in CYP 2A6 activity. The oxidation kinetics for nicotine and cotinine are considered to be different (Hukkanen et al, 2005; Benowitz et al, 2009), and conversion of cotinine to $3 \mathrm{HC}$ is likely more sensitive than altered clearance of nicotine to changes in CYP 2A6 activity. Dempsey et al, 2002 found that cotinine levels were lower in pregnancy despite similar nicotine (smoking) exposure, and that although nicotine clearance increased by $60 \%$ ( $54 \%$ via the cotinine pathway), cotinine clearance increased by $140 \%$. This change was attributed to increased activity of CYP 2A6, which had greater effects on the clearance of cotinine than of nicotine. Similarly, gender and use of oral contraceptives, both thought to effect CYP2A6 activity, had greater impact on cotinine clearance than on the fractional clearance of nicotine to cotinine (Benowitz et al, 2006b).

Although the rewarding or hedonic effects of nicotine appears to be lower in magnitude than those for other drugs of abuse, such as cocaine or heroin, pure nicotine induces significant rewarding effects in humans when delivered rapidly via IV (Soria et al, 1996). These rewarding effects are thought to be mediated by nicotine-induced dopamine release in the reward circuit of the brain (Stein et al, 1998) and to contribute to the reinforcing and addictive properties of nicotine. Our IV nicotine administration procedure, in which nicotine was delivered over $30 \mathrm{~s}$, produced robust subjective rewarding effects in smokers (Sofuoglu et al, 2008). It is important to note that the subjective rewarding effects induced by IV nicotine are less in non-smokers than in smokers (Soria et al, 1996). In contrast, the heart rate increase induced by IV nicotine is greater in non-smokers (Soria et al, 1996). The differences in acute nicotine responses between smokers and non-smokers are likely due to adaptive changes that result from long-term exposure to cigarette smoking.

In our study, smokers in the fourth NMR quartile had greater subjective responses to nicotine for the items that are reflective of reward, including good drug effects, like drug effects, and want more drugs (Fischman and Foltin, 1991; Griffiths et al, 2003). These findings indicate that nicotine is more rewarding in overnight abstinent smokers with faster nicotine metabolism. Many studies have shown that faster nicotine metabolism is associated with greater difficulty in quitting smoking (Lerman et al, 2006; Schnoll et al, 2009). Our findings point to the enhanced rewarding effects of nicotine as a potential contributor to poor success rates of smokers with fast nicotine metabolism. Consistent with this idea, greater positive subjective effects from smoking before an attempt to quit smoking or during a lapse have been predictive of relapse to smoking (Shiffman and Kirchner, 2009; Strong et al, 2011). Shiffman and Kirchner, 2009 used the ecological momentary assessment (EMA) to examine cigarette-by-cigarette satisfaction during an ad lib smoking period in the natural environment of 394 heavy smokers who attempted to quit. Smokers who reported greater satisfaction and pleasure during ad lib smoking were more likely to lapse after quitting. Our findings suggest that enhanced rewarding effects of nicotine combined with greater cigarette craving following abstinence may facilitate relapse in high NMR smokers. These findings further support the need to develop novel treatment approaches for high NMR smokers.

The fourth quartile, compared with other quartiles, was also associated with greater heart rate increases in response to the $0.5 \mathrm{mg}$ but not the $1.0 \mathrm{mg} / 70 \mathrm{~kg}$ dose of IV nicotine. Similarly, for the rating of 'want more drugs' the NMR effects were observed for the $0.5 \mathrm{mg}$ but not the $1.0 \mathrm{mg} / 70 \mathrm{~kg}$ nicotine dose. The reason for the lack of NMR effect on responses to $1 \mathrm{mg} / 70 \mathrm{~kg}$ nicotine dose is not clear. Given that the $0.5 \mathrm{mg}$ nicotine dose always preceded the $1.0 \mathrm{mg}$ nicotine dose, it seems that NMR may have greater influence on the first nicotine response following overnight abstinence. It would be of interest to correlate plasma nicotine levels with acute nicotine response; however, we only collected baseline plasma nicotine levels. Other studies suggest that there could be an accumulation of nicotine levels before the $1.0 \mathrm{mg} / 70 \mathrm{~kg}$ nicotine dose was administered. In a previous study with overnight abstinent smokers, we have shown that $30 \mathrm{~min}$ after $1.0 \mathrm{mg}$ of IV nicotine administration, plasma nicotine levels were about $10 \mathrm{ng} / \mathrm{ml}$ (Sofuoglu et al, 2003). A recent SPECT study demonstrated that following the inhalation of $0.5 \mathrm{mg}$ of nicotine, $55 \%$ of beta $_{2} \mathrm{nAChR}$ were saturated in the brain for 2 to $5 \mathrm{~h}$ (Esterlis et al, 2011). It is possible that the $\mathrm{nAChR}$ receptor occupancy following the $0.5 \mathrm{mg}$ nicotine dose, by inducing tolerance, may result in reduced responses to the $1.0 \mathrm{mg}$ nicotine. Future studies measuring nicotine levels at multiple time points will be needed to compare the dose response curve for different NMR quartiles and to document tolerance.

The possible mechanisms linking NMR to the acute pharmacodynamic effects of nicotine remain to be determined. One possibility is that the rate of nicotine metabolism may influence the amount of nicotine available to bind to nAChRs, which mediate acute nicotine responses. However, faster nicotine metabolism and elimination would be expected to reduce the pharmacological effects of nicotine rather than increase them. Furthermore, given the $2 \mathrm{~h}$ half life of nicotine and that the subjective and cardiovascular effects of nicotine peaked within 3 min after nicotine administration, it is unlikely that faster nicotine elimination would account for these responses (Benowitz et al, 2009). Another possibility is that smokers with slower nicotine metabolism may have delayed recovery of nicotine tolerance as a result of slower decline of brain and plasma nicotine levels. In our study, smokers with high NMR had lower nicotine levels following overnight abstinence. Dempsey et al (2004) showed that smokers with slow nicotine metabolism have a much slower decline of plasma nicotine levels following oral nicotine administration. This may result in attenuated subjective and cardiovascular responses to nicotine following overnight abstinence in smokers with lower NMR compared with those with higher NMR. This possibility warrants future studies examining 
the influence of NMR on nicotine sensitivity following longer durations of smoking abstinence.

Interestingly, although low NMR has been associated with better response to nicotine patches (Schnoll et al, 2009), no such differences in treatment responses have been observed for nicotine spray (Lerman et al, 2006). This may be due to smokers' ability to titrate the nicotine nasal spray, possibly influenced by the rate of nicotine metabolism, compared with the fixed doses in patches. Perhaps, a greater dose of nicotine for those with high NMR may be more effective. Alternatively, pharmacologically slowing the nicotine metabolism rate may increase the treatment response to nicotine. In a proof-of-concept study, Sellers et al (2000) demonstrated that acute treatment with the CYP2A6 inhibitor methoxsalen (10 or $30 \mathrm{mg}$ ) increased plasma nicotine levels following oral nicotine administration $(4 \mathrm{mg})$. Methoxsalen in combination with oral nicotine also reduced ad lib smoking behavior and cigarette craving in smokers, supporting the potential efficacy of methoxsalen to augment the efficacy of nicotine replacement treatment for smoking cessation (Sellers et al, 2000). It is of interest to examine whether nicotine metabolism inhibitors reduce the rewarding effects of nicotine in smokers with fast nicotine metabolism; such an effect may lead to new treatment approaches.

To summarize, higher NMR was associated with a greater craving for cigarettes and a greater reward from IV nicotine following overnight abstinence in smokers, factors that may make quitting more difficult. These findings further support approaches to slow nicotine metabolism as novel treatments for tobacco addiction.

\section{ACKNOWLEDGEMENTS}

We would like to thank Ellen Mitchell, RN, Lance Barnes, Stacy Minnix, and Kathy Barrett for excellent technical assistance. This research was supported by the Veterans Administration Mental Illness Research, Education and Clinical Center (MIRECC) and grants R03-DA 027474, K12 DA000167-20 (AH), and K02-DA021304 (MS) from the National Institute on Drug Abuse (NIDA).

\section{DISCLOSURE}

Dr Sofuoglu serves as an expert witness on behalf of Pfizer in lawsuits related to varenicline. The other authors declare no conflict of interest.

\section{REFERENCES}

al'Absi M, Amunrud T, Wittmers LE (2002). Psychophysiological effects of nicotine abstinence and behavioral challenges in habitual smokers. Pharmacol Biochem Behav 72: 707-716.

Benowitz NL, Hukkanen J, Jacob 3rd P (2009). Nicotine chemistry, metabolism, kinetics and biomarkers. Handb Exp Pharmacol 192: 29-60.

Benowitz NL, Lessov-Schlaggar CN, Swan GE, Jacob 3rd P (2006a). Female sex and oral contraceptive use accelerate nicotine metabolism. Clin Pharmacol Ther 79: 480-488.

Benowitz NL, Jacob 3rd P (2001). Trans-3'-hydroxycotinine: disposition kinetics, effects and plasma levels during cigarette smoking. Br J Clin Pharmacol 51: 53-59.
Benowitz NL, Jacob PI, Ahijevich K, Jarvis MJ, Hall S, LeHouzec J et al (2002). Biochemical verification of tobacco use and cessation. Report from the SRNT Subcommittee on Biochemical Verification. Nicotine Tob Res 4: 149-159.

Benowitz NL, Pomerleau OF, Pomerleau CS, Jacob 3rd P (2003). Nicotine metabolite ratio as a predictor of cigarette consumption. Nicotine Tob Res 5: 621-624.

Benowitz NL, Swan GE, Jacob 3rd P, Lessov-Schlaggar CN, Tyndale RF (2006b). CYP2A6 genotype and the metabolism and disposition kinetics of nicotine. Clin Pharmacol Ther 80: 457-467.

Benowitz NL, Zevin S, Jacob 3rd P (1998). Suppression of nicotine intake during ad libitum cigarette smoking by high-dose transdermal nicotine. J Pharmacol Exp Ther 287: 958-962.

Dallery J, Houtsmuller EJ, Pickworth WB, Stitzer ML (2003). Effects of cigarette nicotine content and smoking pace on subsequent craving and smoking. Psychopharmacology 165: $172-180$

Dempsey D, Jacob 3rd P, Benowitz NL (2002). Accelerated metabolism of nicotine and cotinine in pregnant smokers. J Pharmacol Exp Ther 301: 594-598.

Dempsey D, Tutka P, Jacob 3rd P, Allen F, Schoedel K, Tyndale RF et al (2004). Nicotine metabolite ratio as an index of cytochrome P450 2A6 metabolic activity. Clin Pharmacol Ther 76: 64-72.

Esterlis I, Mitsis EM, Batis JC, Bois F, Picciotto MR, Stiklus SM et al (2011). Brain beta2*-nicotinic acetylcholine receptor occupancy after use of a nicotine inhaler. Int J Neuropsychopharmacol 14: 389-398.

First MB, Spitzer RL, Gibbon M, Williams JBW (1995). Structured Clinical Interview for DSM-IV: Patient Edition. American Psychiatric Press Inc.: Washington, DC.

Fischman MW, Foltin RW (1991). Utility of subjective-effects measurements in assessing abuse liability of drugs in humans. $\mathrm{Br}$ J Addict 86: 1563-1570.

Griffiths RR, Bigelow GE, Ator NA (2003). Principles of initial experimental drug abuse liability assessment in humans. Drug Alcohol Depend 70(3 Suppl): S41-S54.

Hughes JR, Hatsukami D (1986). Signs and symptoms of tobacco withdrawal. Arch Gen Psychiatry 43: 289-294.

Hughes JR, Hatsukami DK (1997). Effects of three doses of transdermal nicotine on post-cessation eating, hunger and weight. J Subst Abuse 9: 151-159.

Hukkanen J, Jacob 3rd P, Benowitz NL (2005). Metabolism and disposition kinetics of nicotine. Pharmacol Rev 57: 79-115.

Jacob 3rd P, Yu L, Duan M, Ramos L, Yturralde O, Benowitz NL (2011). Determination of the nicotine metabolites cotinine and trans-3'-hydroxycotinine in biologic fluids of smokers and nonsmokers using liquid chromatography-tandem mass spectrometry: biomarkers for tobacco smoke exposure and for phenotyping cytochrome P450 2A6 activity. J chromatogr B, Analyt Technol Biomed Life Sci 879: 267-276.

Kandel DB, Hu MC, Schaffran C, Udry JR, Benowitz NL (2007). Urine nicotine metabolites and smoking behavior in a multiracial/multiethnic national sample of young adults. $A m \mathrm{~J}$ Epidemiol 165: 901-910.

Lea RA, Dickson S, Benowitz NL (2006). Within-subject variation of the salivary $3 \mathrm{HC} / \mathrm{COT}$ ratio in regular daily smokers: prospects for estimating CYP2A6 enzyme activity in large-scale surveys of nicotine metabolic rate. J Analyt toxicol 30: 386-389.

Johnstone E, Benowitz N, Cargill A, Jacob R, Hinks L, Day I et al (2006). Determinants of the rate of nicotine metabolism and effects on smoking behavior. Clin Pharmacol Ther 80: 319-330.

Lerman C, Tyndale R, Patterson F, Wileyto EP, Shields PG, Pinto A et al (2006). Nicotine metabolite ratio predicts efficacy of transdermal nicotine for smoking cessation. Clin Pharmacol Ther 79: 600-608. 
Levi M, Dempsey DA, Benowitz NL, Sheiner LB (2007). Prediction methods for nicotine clearance using cotinine and 3-hydroxycotinine spot saliva samples II. Model application. J Pharmacokinet Pharmacodyn 34: 23-34.

Malaiyandi V, Lerman C, Benowitz NL, Jepson C, Patterson F, Tyndale RF (2006). Impact of CYP2A6 genotype on pretreatment smoking behaviour and nicotine levels from and usage of nicotine replacement therapy. Mol psychiatry 11: 400-409.

Mendelson JH, Sholar MB, Goletiani N, Siegel AJ, Mello NK (2005). Effects of low- and high-nicotine cigarette smoking on mood states and the HPA axis in men. Neuropsychopharmacology 30: 1751-1763.

Mooney ME, Li ZZ, Murphy SE, Pentel PR, Le C, Hatsukami DK (2008). Stability of the nicotine metabolite ratio in ad libitum and reducing smokers. Cancer Epidemiol Biomarkers Prev 17: $1396-1400$

Mwenifumbo JC, Sellers EM, Tyndale RF (2007). Nicotine metabolism and CYP2A6 activity in a population of black African descent: impact of gender and light smoking. Drug Alcohol depend 89: 24-33.

Mwenifumbo JC, Tyndale RF (2009). Molecular genetics of nicotine metabolism. Handbook Exp pharmacol 192): 235-259.

Newhouse PA, Sunderland T, Narang PK, Mellow AM, Fertig JB, Lawlor BA et al (1990). Neuroendocrine, physiologic, and behavioral responses following intravenous nicotine in nonsmoking healthy volunteers and in patients with Alzheimer's disease. Psychoneuroendocrinology 15: 471-484.

Patterson F, Schnoll RA, Wileyto EP, Pinto A, Epstein LH, Shields $\mathrm{PG}$ et al (2008). Toward personalized therapy for smoking cessation: a randomized placebo-controlled trial of bupropion. Clin Pharmacol Ther 84: 320-325.

Pickworth WB, Fant RV (1998). Endocrine effects of nicotine administration, tobacco and other drug withdrawal in humans. Psychoneuroendocrinology 23: 131-141.

Rao Y, Hoffmann E, Zia M, Bodin L, Zeman M, Sellers EM et al (2000). Duplications and defects in the CYP2A6 gene: identification, genotyping, and in vivo effects on smoking. Mol Pharmacol 58: 747-755.

Rubinstein ML, Benowitz NL, Auerback GM, Moscicki AB (2008). Rate of nicotine metabolism and withdrawal symptoms in adolescent light smokers. Pediatrics 122: e643-e647.

SAS Institute Inc (2007). The SAS System for Windows, 9.1.3. SAS Institute Inc: Cary, NC.

Schnoll RA, Patteson F, Wileyto EP, Tyndale RF, Benowitz N, Lerman C (2009). Nicotine metabolic rate predicts successful smoking cessation with transdermal nicotine: a validation study. Pharmacol Biochem Behav 92: 6-11.
Sellers EM, Kaplan HL, Tyndale RF (2000). Inhibition of cytochrome P450 2A6 increases nicotine's oral bioavailability and decreases smoking. Clin Pharmacol Ther 68: 35-43.

Shiffman S, Kirchner TR (2009). Cigarette-by-cigarette satisfaction during ad libitum smoking. J Abnorm Psychol 118: 348-359.

Sofuoglu M, Babb D, Hatsukami DK (2003). Labetalol treatment enhances the attenuation of tobacco withdrawal symptoms by nicotine in abstinent smokers. Nicotine Tob Res 5: 947-953.

Sofuoglu M, Mouratidis M, Yoo S, Culligan K, Kosten T (2005). Effects of tiagabine in combination with intravenous nicotine in overnight abstinent smokers. Psychopharmacology 181: 504-510.

Sofuoglu M, Poling J, Mouratidis M, Kosten T (2006). Effects of topiramate in combination with intravenous nicotine in overnight abstinent smokers. Psychopharmacology 184: 645-651.

Sofuoglu M, Waters AJ, Mooney M, O’Malley SS (2009). Minocycline reduced craving for cigarettes but did not affect smoking or intravenous nicotine responses in humans. Pharmacol Biochem Behav 92: 135-140.

Sofuoglu M, Yoo S, Hill KP, Mooney M (2008). Self-administration of intravenous nicotine in male and female cigarette smokers. Neuropsychopharmacology 33: 715-720.

Soria R, Stapleton JM, Gilson SF, Sampson-Cone A, Henningfield JE, London ED (1996). Subjective and cardiovascular effects of intravenous nicotine in smokers and non-smokers. Psychopharmacology 128: 221-226.

Stein EA, Pankiewicz J, Harsch HH, Cho JK, Fuller SA, Hoffmann RG et al (1998). Nicotine-induced limbic cortical activation in the human brain: a functional MRI study. Am J Psychiatry 155: 1009-1015.

Strasser AA, Benowitz NL, Pinto AG, Tang KZ, Hecht SS, Carmella SG et al (2011). Nicotine metabolite ratio predicts smoking topography and carcinogen biomarker level. Cancer Epidemiol Biomarkers Prev 20: 234-238.

Strong DR, Leventhal AM, Evatt DP, Haber S, Greenberg BD, Abrams D et al (2011). Positive reactions to tobacco predict relapse after cessation. J Abnorm Psychol 120: 999-1005.

Swan GE, Lessov-Schlaggar CN, Bergen AW, He Y, Tyndale RF, Benowitz NL (2009). Genetic and environmental influences on the ratio of 3 'hydroxycotinine to cotinine in plasma and urine. Pharmacogenet Genomics 19: 388-398.

Wassenaar CA, Dong Q, Wei Q, Amos CI, Spitz MR, Tyndale RF (2011). Relationship Between CYP2A6 and CHRNA5-CHRNA3CHRNB4 Variation and Smoking Behaviors and Lung Cancer Risk. J Natl Cancer Inst 103: 1342-1346.

West O, Hajek P, McRobbie H (2011). Systematic review of the relationship between the 3-hydroxycotinine/cotinine ratio and cigarette dependence. Psychopharmacology 218: 313-313. 